\title{
Reproductive Conflict and the Costs of Social Status in Cooperatively Breeding Vertebrates
}

\author{
Dustin R. Rubenstein ${ }^{1, *}$ and Sheng-Feng Shen ${ }^{2}$ \\ 1. Department of Integrative Biology and Museum of Vertebrate Zoology, University of California, Berkeley, California 94720; \\ 2. Department of Neurobiology and Behavior, Cornell University, Ithaca, New York 14853 \\ Submitted September 29, 2008; Accepted December 8, 2008; Electronically published March 18, 2009 \\ Online enhancement: appendix.
}

\begin{abstract}
Conflict over reproduction is an inherent part of group living. In many social vertebrates, conflict may be reflected as allostatic load, or the costs of social status and dominance rank, which may be quantified by measuring glucocorticoid stress hormones. Here, we develop the first quantitative model of allostatic load based on the tug-of-war model of reproductive skew to generate insights into the mechanisms underlying reproductive conflict in cooperative breeders and to determine whether glucocorticoids can be used to assess conflict levels in group-living vertebrates. It predicts that subordinates have higher allostatic loads than dominants under most conditions, but when body condition is lower in dominants than in subordinates, dominants experience higher allostatic load. Group structure is also important, as dominants generally have higher allostatic loads than subordinates when there is a large number of subordinates in the group, but this cost can be reduced by increasing the number of dominants, as in plural breeding societies. Using glucocorticoid data from cooperatively breeding superb starlings Lamprotornis superbus, we found empirical support for both predictions. Our model is useful for understanding how the costs of social status influence reproductive sharing, and it suggests that glucocorticoids can be used to examine reproductive conflict and cooperation in social species.
\end{abstract}

Keywords: allostasis, allostatic load, reproductive skew, dominance rank, cooperative breeding, glucocorticoids.

\section{Introduction}

Living in cooperatively breeding groups, where more than two individuals care for young, provides a variety of reproductive and survival benefits to nonbreeding group members (Brown 1987; Cockburn 1998). Despite the inclusive fitness and other benefits afforded to nonbreeders for remaining in a group, conflict over direct reproduction is an inherent part of most groups where there are breeding opportunities for subordinate individuals (Emlen 1982,

\footnotetext{
* Corresponding author; e-mail: drubenstein@berkeley.edu.

Am. Nat. 2009. Vol. 173, pp. 650-661. (c) 2009 by The University of Chicago. 0003-0147/2009/17305-50767\$15.00. All rights reserved. DOI: $10.1086 / 597606$
}

1995; Vehrencamp 1983b). Reproductive or social suppression of subordinate individuals by dominant individuals has long been suggested to explain why some group members in social species fail to breed (Wasser and Barash 1983). However, because overt aggressive interactions between group members are rarely observed in many cooperatively breeding species, numerous studies have tried to test the social suppression hypothesis by measuring glucocorticoid (GC) stress hormones in dominant and subordinate individuals (reviewed in Creel 2001; Goymann and Wingfield 2004). Because subordinate individuals tend to have higher GC levels than dominant individuals in most noncooperatively breeding species (Creel 2001), subordinate helpers in most cooperatively breeding species would be expected to have higher GC levels than dominant breeders if social subordination explains the adoption of different breeding roles (Mays et al. 1991; Schoech et al. 1991, 1997; Wingfield et al. 1991). However, evidence for GC-related social suppression of subordinate reproduction in free-living vertebrates is lacking (reviewed in Creel 2001; Goymann and Wingfield 2004) in all but a few species (Faulkes and Abbott 1997; Young et al. 2006), making the interpretation of GC patterns difficult and their utility in mechanistic studies of social behavior questionable.

In most vertebrates, GCs regulate a variety of physiological processes and are released in response to predictable and unpredictable events in the life cycle, including both transitory and more permanent environmental (e.g., seasonality, weather events) and social (e.g., social status) factors (McEwen and Wingfield 2003). The cumulative result of these factors on homeostasis and GC physiology, or the energetic costs of dealing with predictable and unpredictable daily events, is termed "allostatic load" (McEwen and Wingfield 2003). For individuals living in social groups, allostatic load has been used to describe the costs associated with social status, dominance rank, and being part of a stable social unit (Goymann and Wingfield 2004; Rubenstein 2007a). Thus, for dominant individuals, allostatic load refers to the costs of acquiring and main- 
taining dominance rank, whereas for subordinate individuals, allostatic load refers to the costs of trying to attain higher rank and coping with physical or psychological harassment by dominant individuals (Goymann and Wingfield 2004). In many social carnivores, dominant breeders typically have higher levels of GCs (Creel 2001, 2005; Creel and Creel 2002; Sands and Creel 2004) and higher allostatic loads (Goymann and Wingfield 2004) than subordinate helpers. However, across broad phylogenetic scales, GC patterns and breeding roles in cooperatively breeding vertebrates are related to amounts of socially induced allostatic load such that dominant breeders have higher GC levels and allostatic loads than do subordinate helpers in some species but lower levels in others (McEwen and Wingfield 2003; Goymann and Wingfield 2004). Additionally, in some species like the cooperatively breeding superb starling Lamprotornis superbus, patterns of GC levels and allostatic load may vary from year to year and are related to environmental conditions such that subordinate individuals have higher levels of GCs in drier years but lower levels of GCs in wetter years (Rubenstein 2007a). Together, these studies emphasize how both social and environmental stressors can influence GC levels and allostatic load in group-living vertebrates. Determining whether allostatic load theory can explain GC patterns and the adoption of different breeding roles in social vertebrates requires developing theoretical predictions that account for the dual influences of social and environmental stressors on GC level and allostatic load and that can be tested in free-living species.

Here, we provide the first quantitative model of allostatic load by employing the tug-of-war model of reproductive skew (Reeve et al. 1998). Although most reproductive skew models predict the optimal degree of reproductive sharing in group-living animals or the reproductive benefits of living in groups (Johnstone 2000), we model how social and environmental factors influence one of the primary costs of group living: allostatic load. We describe an $n$-player game theory model that provides important insights into the mechanisms underlying reproductive conflict and the costs of social status in cooperatively breeding groups. Because relative GC levels are positively correlated with relative levels of allostatic load across a variety of taxa (Goymann and Wingfield 2004), we develop a series of testable predictions to determine the utility of GC levels for studying reproductive conflict in social vertebrates. We then test the two key predictions from our model relating allostatic load to body condition and group structure using multiyear data from cooperatively breeding superb starlings. Additionally, we discuss how this model might apply to different types of animal societies and explain alternative forms of sociality where reproductive sharing and the types of dominance hierar- chies vary. Ultimately, this study illustrates the importance of considering the direct costs of dominance interactions in reproductive skew models, and it provides a robust theoretical framework to predict how studies of GCs and other aspects of vertebrate physiology can be used to examine reproductive conflict and cooperation in social species.

\section{The Allostatic Load Model: Tug-of-War Revisited}

Reproductive skew theory seeks to describe the variation in reproductive partitioning among group members (Vehrencamp 1983a, 1983b). Models of reproductive skew can be classified into two groups: transactional models and tug-of-war models. In transactional models, group members yield shares of reproduction to each other in return for cooperation, whereas in tug-of-war models, group members invest group resources in a tug-of-war over their reproductive shares (Johnstone 2000; Reeve and Keller 2001; Reeve and Shen 2006). It is assumed in transactional models that either the dominant individual or the subordinate individual is fully in control of the distribution of reproduction. Therefore, transactional models are only suitable for modeling potential conflict (versus actual conflict), because if one individual has complete control over group resources, no actual conflict would be expected to occur. On the other hand, the distribution of reproduction in tug-of-war models is assumed to be determined by costly competition (Reeve and Keller 2001). We thus chose to base our model on the tug-of-war model of reproductive skew (Reeve et al. 1998; see also Frank 1995) because it is an appropriate place to start modeling the costs of conflict in cooperative species. In the original tug-of-war model, costs are considered only in terms of group resources, and such costs do not impact an individual's future reproductive success (Reeve et al. 1998). In our model, we extend the idea of personal costs and assume that being part of a group and competing for breeding opportunities (i.e., partaking in a tug-of-war) has an additional cost in terms of a reduction in future reproductive success.

We extend the basic tug-of-war model (Reeve et al. 1998 ) to an $n$-player game (i.e., $\geq 2$ players) with two different classes of group members, alphas and betas. We assume that alphas and betas can compete freely over a share of reproduction and that individuals in the same class are homogeneous, or that they exhibit the same competitive abilities. Therefore, between-rank competition (i.e., alpha vs. beta) is greater than within-rank competition (i.e., alpha vs. alpha or beta vs. beta) in our model. Allowing for individual differences within classes in our model is mathematically equivalent to having more classes of individuals, but for simplicity, we only consider two classes here. The terms $n_{\alpha}$ and $n_{\beta}$ describe the numbers 
of alphas and betas in the group, respectively. We describe the following model in terms of a single alpha and a single beta, but later we present results with varying numbers of alphas and betas.

Alpha and beta vary in their relative competitive abilities (b). When $b$ is equal to 1 , the competitive ability of beta is equal to that of alpha, and as $b$ declines from 1 , alpha becomes increasingly more competitive than beta. We assume that, for each individual, there is a trade-off between contributing to the group total reproduction $\left(i_{\alpha}+i_{\beta}\right.$, where $i_{\alpha}$ and $i_{\beta}$ are the contributions of alpha and beta, respectively) and fighting for a personal share of the reproduction ( $x$ and $y$ for alpha and beta, respectively). The $x$ and $y$ variables can be viewed as each individual's level of selfish effort because the more effort ( $x$ or $y$ ) that is invested in the costly competition, the higher the individual's personal share but the lower the group's total reproduction $\left(i_{\alpha}+i_{\beta}\right)$. More explicitly, alpha can freely decide what fraction $x$ of its total effort to put into the costly competition with beta versus investing in the group total reproduction $\left(i_{\alpha}=1-x\right)$. Likewise, beta can decide what fraction of $y$ and $i_{\beta}=1-y$ to invest in competition and the group reproduction, respectively. It is important to point out, however, that in our game theoretical model, the levels of selfish efforts of alpha and beta are evolutionarily stable. That is, any level of selfish effort that one player expends is always the optimal response to another player's level of selfish effort. In other words, $x$ and $y$ are linked according to the Nash equilibrium, and these variable values cannot be chosen arbitrarily. Therefore, the fraction of group total reproduction going to alpha is $x /\left(n_{\alpha} x+n_{\beta} b y\right)$, the fraction going to beta is $b y /\left(n_{\alpha} x+\right.$ $n_{\beta} b y$ ), and the group total reproduction is equal to

$$
g\left(n_{\alpha} i_{\alpha}+n_{\beta} i_{\beta}\right)^{z}=g\left[\left(n_{\alpha}+n_{\beta}\right)-n_{\alpha} x-n_{\beta} y\right]^{z},
$$

where $g$ and $z$ are scaling factors used to describe the level of group benefit generated by each individual's contribution. Specifically, $z$ describes the shape (e.g., concave or convex) of group productivity in relation to an individual's contribution, and $g$ indicates the magnitude of the cooperative benefit. We constrain $z$ to be less than 1 to create a diminishing-returns function on group productivity. That is, when $z$ approaches 0 , each individual's contribution $\left(i_{\alpha}\right.$ or $i_{\beta}$ ) does not influence the magnitude of the group productivity much, whereas when $z$ approaches 1 , group productivity increases linearly as each individual's contribution to the group output increases.

When two individuals engage in the costly tug-of-war process, we assume that each incurs a cost that is directly related to the intensity of the conflict. This personal cost associated with living in a group is the same as the cost of social status, or allostatic load. We follow the definition of Goymann and Wingfield (2004) and assume that the cost to alpha associated with engaging in aggressive interactions with beta is the same as the cost of acquiring and maintaining its higher dominance rank, whereas the cost to beta of fighting with alpha is the same as the cost of attempting to attain higher rank and of coping with aggression (either physical or psychological) by the more dominant alpha. Thus, this personal cost is a product of both the aggressive effort expended and received during within-group social interactions.

We use the accelerating functions $d_{\alpha} e x^{2}$ and $d_{\beta} e y^{2}$ for alpha and beta, respectively, to describe the nature of this personal cost, or the level of allostatic load. Because allostatic load is the cumulative result of both social and environmental stressors (McEwen and Wingfield 2003), we explicitly built our allostatic load term to be a function of both levels of selfish effort ( $x$ or $y$ ) and body condition $(d)$, which are related to social and environmental stressors, respectively. However, body condition need not only be related to environmental stressors: it can be influenced by the accumulation of both environmental and social stressors, and our model accounts for this.

Recall that the cost of selfish effort refers to the player's cost in terms of reduced reproduction while the player is fighting for a share of reproduction and trying to maintain its rank. However, because we assume that competing for a share of reproduction is physiologically costly, selfish effort also causes the cost of the recipient's physiological response to aggression. Therefore, allostatic load $\left(d_{\alpha} e x^{2}\right.$ or $\left.d_{\beta} e y^{2}\right)$ is directly proportional to $x$ and $y$, or the levels of selfish effort for alpha and beta, respectively, where $e$ is a scaling factor representing the magnitudes of the trade-off between current and future reproduction. When $e$ is small, the current breeding event is less costly than future breeding events (i.e., a trade-off between current and future reproduction), a scenario that is likely to apply to longlived species or to those that breed multiply over the course of their lives, such as most cooperatively breeding vertebrates. We use accelerating cost functions (i.e., raised to the power of 2) to represent the personal costs of investing in current breeding competition, because this is a standard assumption in life-history evolution theory (Trivers 1972; Charnov and Krebs 1974). Personal cost is defined as the loss of future fitness, and therefore it is equivalent to a convex-down relationship between investment in the current brood and in adult survivorship. In other words, investing too much in survival only or current reproduction only yields diminishing returns in fitness. Although we use this particular power function in our model, the results are qualitatively similar if other accelerating functions are used. As levels of selfish effort rise and fighting in the group intensifies, the costs of social status (i.e., allostatic load) increases proportionally. 
Allostatic load is also directly proportional to the body conditions of alpha and beta, $d_{\alpha}$ and $d_{\beta}$, respectively. We define body condition simply as an estimate of nutritional state (i.e., relative "fatness"; sensu Jakob et al. 1996). Smaller values of $d$ indicate that an individual is in better condition, whereas larger values of $d$ indicate that an individual is in poorer condition. We assume that body condition influences the fitness payoffs for alpha and beta by affecting the costs associated with each individual's level of selfish effort; individuals in better condition suffer fewer costs resulting from their level of selfish effort, and vice versa. In other words, environmental stressors influence allostatic load by indirectly affecting competitive interactions among group members.

Ultimately, we can obtain alpha's fitness function, $w_{\alpha}$, and beta's fitness function, $w_{\beta}$, as follows:

$$
\begin{aligned}
& w_{\alpha}=g\left[\left(n_{\alpha}+n_{\beta}\right)-n_{\alpha} x-n_{\beta} y\right]^{z} \frac{x}{n_{\alpha} x+n_{\beta} b y}-d_{\alpha} e x^{2}, \\
& w_{\beta}=g\left[\left(n_{\alpha}+n_{\beta}\right)-n_{\alpha} x-n_{\beta} y\right]^{z} \frac{b y}{n_{\alpha} x+n_{\beta} b y}-d_{\beta} e y^{2} .
\end{aligned}
$$

The inclusive fitnesses of alpha and beta are then

$$
\begin{aligned}
& W_{\alpha}=w_{\alpha}+\left(n_{\alpha}-1\right) r_{\alpha \alpha} w_{\alpha}+n_{\beta} r_{\alpha \beta} w_{\beta}, \\
& W_{\beta}=w_{\beta}+\left(n_{\beta}-1\right) r_{\beta \beta} w_{\beta}+n_{\alpha} r_{\alpha \beta} w_{\alpha},
\end{aligned}
$$

respectively, where $r_{\alpha \alpha}$ is the genetic relatedness between alphas, $r_{\beta \beta}$ is the genetic relatedness between betas, and $r_{\alpha \beta}$ is the genetic relatedness between alpha and beta. Thus, there are two targets of selection: (1) beta's selfish effort in the tug-of-war $(y)$, and (2) alpha's selfish effort in the tug-of-war $(x)$. Using numerical solutions, we solve for the evolutionarily stable values of these decision variables by simultaneously solving the two equations:

$$
\begin{gathered}
\frac{\partial W_{\alpha}}{\partial x}=0, \\
\frac{\partial W_{\beta}}{\partial y}=0,
\end{gathered}
$$

where $x=x^{*}$ and $y=y^{*}$.

\section{Predictions from the Two-Player Version of the Model}

Recall that we define allostatic load in our model $\left(d_{\alpha} e x^{2}\right.$ or $d_{\beta} e y^{2}$ for alpha or beta, respectively) as being equivalent to the personal cost of selfish effort, which is directly modulated by both levels of selfish effort ( $x$ and $y$ ) and body condition $\left(d_{\alpha}\right.$ and $\left.d_{\beta}\right)$. Similar to our term for relative competitive ability, it is easier to conceptualize the body conditions of alpha and beta in relative terms (i.e., relative body condition of alpha, $\left.d_{\alpha} / d_{\beta}\right)$. When $d_{\alpha} / d_{\beta}<1$, alpha is in better condition and is more efficient in exerting selfish effort (i.e., suffers less personal cost), and when $d_{\alpha} / d_{\beta}>$ 1 , beta is in better condition and is more efficient at exerting selfish effort.

The two-player version of the model with one alpha and one beta predicts that relative body condition of alpha can differentially affect the relative allostatic load of alpha such that, under certain conditions, alpha can have a higher allostatic load than beta, and vice versa. That is, beta's allostatic load is greater than alpha's when beta is in poorer condition, whereas alpha's allostatic load is greater than beta's when alpha is in poorer condition (fig. $1 A$ ). Thus, although the relative allostatic load of alpha will typically be less than 1 , under some conditions it will be greater than 1 (fig. $1 B$ ). This occurs because, even when alpha and beta have similar body conditions $\left(d_{\alpha} / d_{\beta}=1\right)$, the selfish effort of beta is always higher than that of alpha (assuming beta is less competitive than alpha, or $b<1$ ). Thus, beta's inefficiency to convert selfish effort to compete for resources forces beta to invest a higher level of selfish effort than alpha despite their similar body conditions (for details, see Reeve et al. 1998). Although this result is somewhat intuitive given the way we built our allostatic load term, it should be noted that there are conditions under which alpha's and beta's allostatic load curves will not cross (see the appendix and fig. A1 in the online edition of the American Naturalist). This occurs because the allostatic loads of alpha and beta are modulated by both social and environmental factors in our model, and thus poor condition alone does not always lead to higher allostatic loads. Also of note is the finding that the switch point between when either alpha or beta has higher allostatic load is greater than 1 .

Allostatic load is also indirectly influenced by two additional parameters in our model: relative competitive ability $(b)$ and genetic relatedness $(r)$. The model predicts that the allostatic loads of alpha and beta increase as their relative competitive abilities become more similar (fig. 2 A). This occurs because, when beta's competitive ability is similar to alpha's, it becomes beneficial for beta to exert a greater level of selfish effort, and in response, alpha must also invest greater selfish effort as the conflict escalates. This result is similar to many game-theoretical models of conflict (e.g., Maynard Smith and Parker 1976; Parker and Rubenstein 1981). However, beta will always have a higher allostatic load than alpha (assuming $d_{\alpha} / d_{\beta} \leq 1$ ) because beta, by definition, is less efficient in costly competition $(b<1)$ and would exert a higher level of selfish effort in competing for group resources than alpha. Thus, the rel- 
A

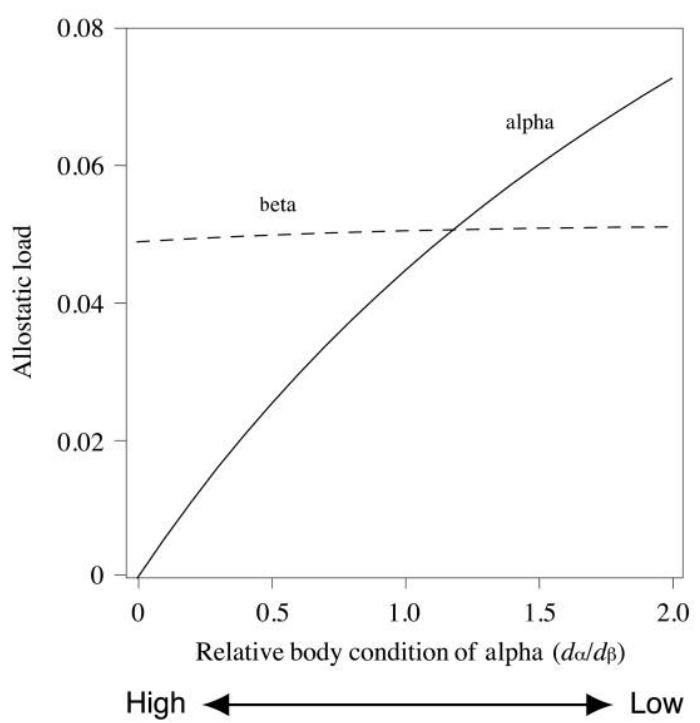

B

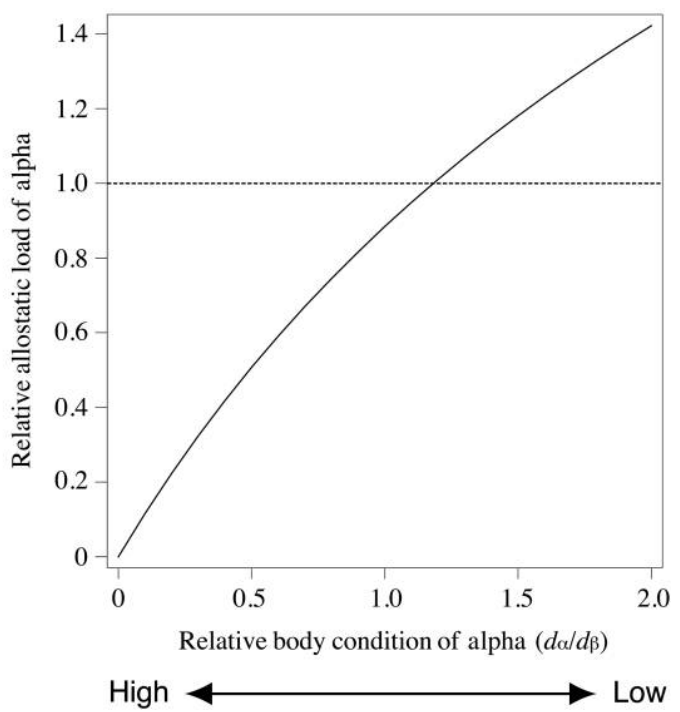

Figure 1: Allostatic loads of alpha and beta $(A)$ and relative allostatic load of alpha in relation to relative body condition of alpha $(B)$. Relative allostatic load of alpha was calculated as the ratio of alpha's allostatic load to beta's allostatic load, and relative body condition of alpha was calculated as the ratio of alpha's body condition to beta's body condition. The dashed line indicates beta's level of allostatic load, whereas the solid line indicates alpha's level of allostatic load. The dotted line indicates a relative allostatic load for alpha of 1 . For all models, relatedness $(r)$ was 0.25 , the relative competitive ability $(b)$ was 0.8 , and the scaling factors $g, z$, and $e$ were $2,0.7$, and 0.3 , respectively.

ative allostatic load of alpha will always be less than 1 (fig. $2 B$ ). Our model also predicts that the allostatic loads of alpha and beta decrease with increasing genetic relatedness (fig. 2C). When alpha and beta are more closely related to each other, their fitness expressions are more similar. Therefore, because the costs of competing (i.e., levels of selfish effort) are lower, the allostatic loads of both alpha and beta will also be lower. Again, beta will always have a higher allostatic load than alpha (assuming $r<1$ ) because of beta's inefficiency to convert personal resources into selfish effort, as described previously. Thus, the relative allostatic load of alpha will always be less than 1 (fig. $2 D)$. Finally, our model is relatively insensitive to the three scaling factors $(z, g$, and $e)$. That is, beta will always have higher allostatic load than alpha for all values (greater than 0 ) of each parameter (see the appendix and fig. A2).

\section{Predictions from the $n$-Player Version of the Model}

Up to this point, our model has explored the most simple case with only two players, a single alpha and a single beta. However, varying the number of alphas and/or betas $\left(n_{\alpha}\right.$ and $n_{\beta}$, respectively) in the group has important implications for understanding how this type of model applies to different types of social taxa where group sizes, group structures, and dominance hierarchies may vary (Stacey and Koenig 1990; Solomon and French 1997). Although our model assumes a relatively simple hierarchy with greater between-rank than within-rank competition, other types of hierarchies could be modeled in a similar manner by extending our model with additional ranks (e.g., alpha, beta, gamma, etc.) and/or by using a neighbor-modulated fitness approach (Hamilton 1964; Taylor and Frank 1996). When expanding the two-player model to an $n$-player model, we find that as we increase the number of alphas and betas in the group, the allostatic loads of both alpha and beta covary with the number of individuals in each class. Alpha's allostatic load increases as the number of betas in the group increases, but beta's allostatic load decreases as the number of betas increases (fig. 3). As a result, the relative allostatic load of alpha increases as the number of betas in the group increases (fig. $4 A$ ), but the relative allostatic load of alpha decreases as the number of alphas in the group increases (fig. 4B). Therefore, alphas generally have a higher allostatic load than betas when there is a large number of betas in the group, but this cost can be reduced by increasing the number of alphas in the group (as in plural breeding societies). It is important to note that the absolute number of alphas or betas in the group is less important than the ratio of alphas to betas in the group. For example, the curves for the equation with one alpha and one beta in figure $4 A$ and $4 B$ are identical to 
A

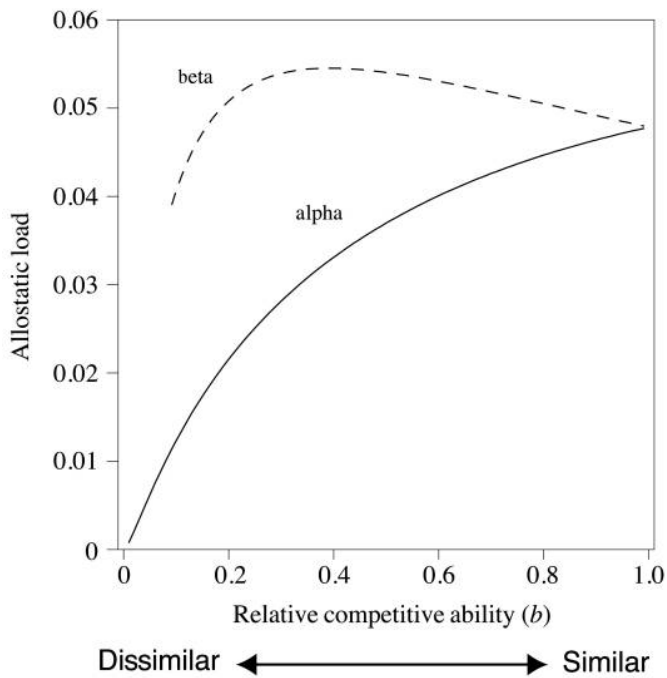

C

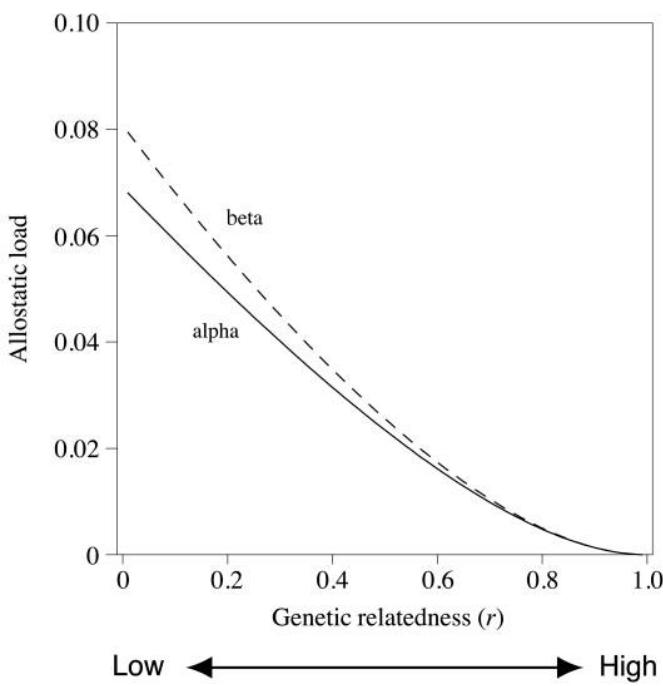

B

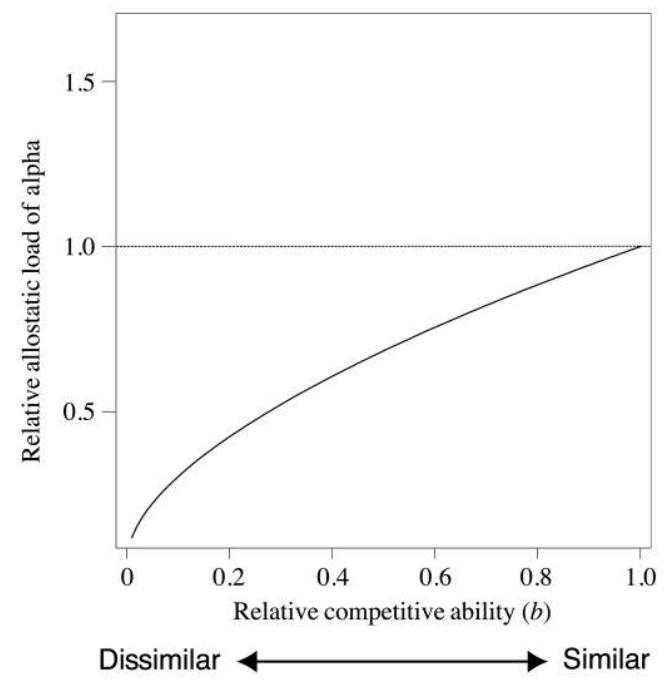

D

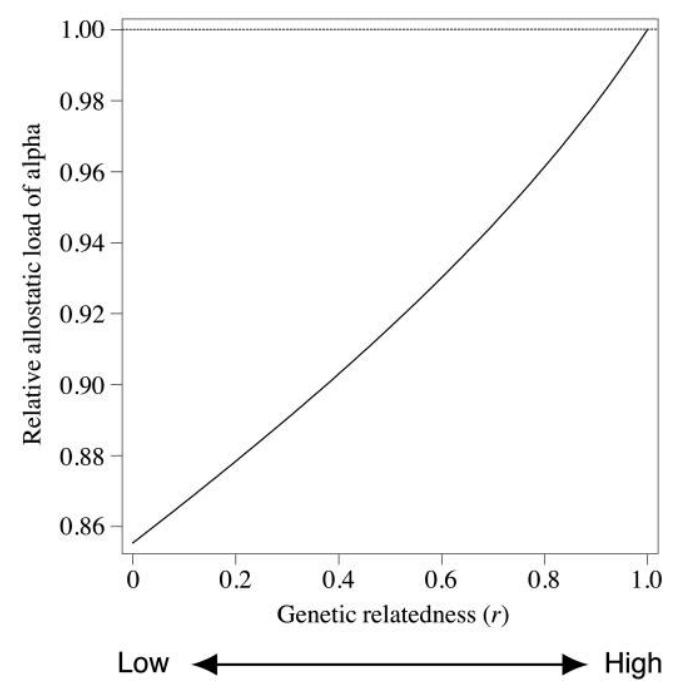

Figure 2: Allostatic loads of alpha and beta $(A, C)$ and relative allostatic load of alpha $(B, D)$ as a function of relative competitive ability $(b)$ and genetic relatedness $(r)$. Relative allostatic load of alpha was calculated as the ratio of alpha's allostatic load to beta's allostatic load. Dashed lines indicate beta's level of allostatic load, whereas solid lines indicate alpha's level of allostatic load. Dotted lines indicate a relative allostatic load for alpha of 1 . For all models, relative body condition of alpha $\left(d_{\alpha} / d_{\beta}\right)$ was 1 , and the scaling factors $g$, $z$, and $e$ were $2,0.7$, and 0.3 , respectively. Additionally, relatedness $(r)$ was 0.25 in models $A$ and $B$, and the relative competitive ability $(b)$ was 0.8 in models $C$ and $D$.

the curve for the equation with two alphas and two betas in figure $4 C$; both are at a $1: 1$ ratio. Additionally, body condition is still extremely important in the $n$-player model, and modulating the relative body condition of alpha ( $X$-axis in fig. 4) can simultaneously affect the relative allostatic load of alpha (fig. 4). Thus, both body condition and group structure can influence the allostatic loads of alpha and beta.

\section{Summarizing the Key Predictions of the Allostatic Load Model}

Our model makes two key predictions. First, it predicts that, in a two-player game, allostatic load-or the costs of social status-will always be higher in subordinate individuals than in dominant individuals, except in some cases when there are differences in body condition. That is, only 


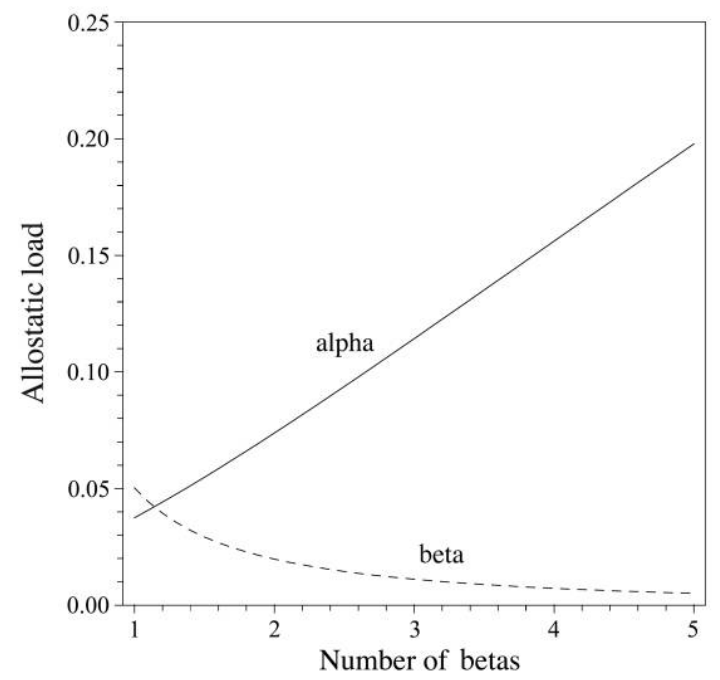

B

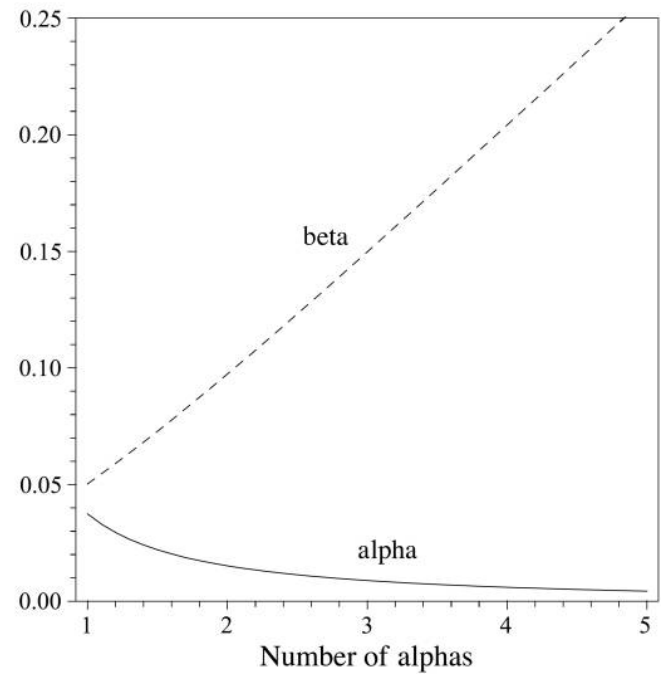

Figure 3: Allostatic loads of alpha and beta in groups with $A$, one alpha and a variable number of betas and $B$, one beta and a variable number of alphas. The dashed line indicates beta's level of allostatic load, whereas the solid line indicates alpha's level of allostatic load. For all models, relatedness $(r)$ was 0.25 , the relative competitive ability $(b)$ was 0.8 , and the scaling factors $g, z$, and $e$ were $2,0.7$, and 0.3 , respectively.

when the dominant individual is in poorer body condition than the subordinate individual will the dominant individual possibly experience higher allostatic load. Both relative competitive abilities and relatedness impact the magnitudes of individual allostatic loads, but they do not alter the relative level of alpha's allostatic load; subordinate individuals will always have higher levels of allostatic load than dominant individuals for all states of these parameters (assuming $d_{\alpha} / d_{\beta} \leq 1$ ). Second, when our model is expanded from a simple two-player game to an $n$-player game where the number of alphas and/or betas can vary, it predicts that not only is body condition still important in influencing allostatic load, but group structure, or the ratio of alphas to betas in the group, is also important. That is, relative allostatic load of alpha increases as the number of betas in the group increases, but it decreases as the number of alphas in the group increases. Thus, alphas will generally have higher allostatic loads than betas when there is a large number of betas in the group, but this cost can be reduced by increasing the number of alphas in the group.

\section{An Empirical Test of the Allostatic Load Model}

There are few published multiyear data from the same individuals of body condition and corresponding GC levels (a proxy for allostatic load; Goymann and Wingfield 2004) in dominant and subordinate group members of cooperatively breeding species. However, we used previously published data from plural breeding superb starlings $(\mathrm{Ru}-$ benstein $2007 a$ ) to test the two key predictions from our model. Superb starlings live in large social groups with multiple breeders and helpers of both sexes (Rubenstein $2007 a, 2007 b$ ). Because breeders are socially dominant to helpers in this system (Rubenstein 2007a), we define alphas as breeders and betas as helpers. In superb starlings, levels of the GC corticosterone were related to year-toyear variation in prebreeding rainfall in subordinate helpers, but not in dominant breeders, whereas standardized body mass (body condition) in breeders, but not in helpers, was related to the interannual variation in rainfall (Rubenstein 2007a). The relationship between standardized body mass and rainfall was such that breeders had higher standardized body masses in drier years and lower standardized body masses in wetter years (Rubenstein $2007 a, 2007 b$ ). Although gaining body mass during harsh conditions seems to be counterintuitive, it is a well-documented response to unpredictable food supply in European starlings Sturnus vulgaris, and it may be linked to reduced activity and metabolic expenditure, as well as an increase in fat stores, to endure the period of food shortage (Witter et al. 1995; Cuthill et al. 2000; Buchanan et al. 2003). In superb starlings, these seemingly counterintuitive patterns of body-mass change with rainfall have been directly linked to fitness gains in terms of how mothers bias offspring sex ratio according to the amount of rainfall (Rubenstein 2007b).

We continuously monitored seven to nine social groups 
A

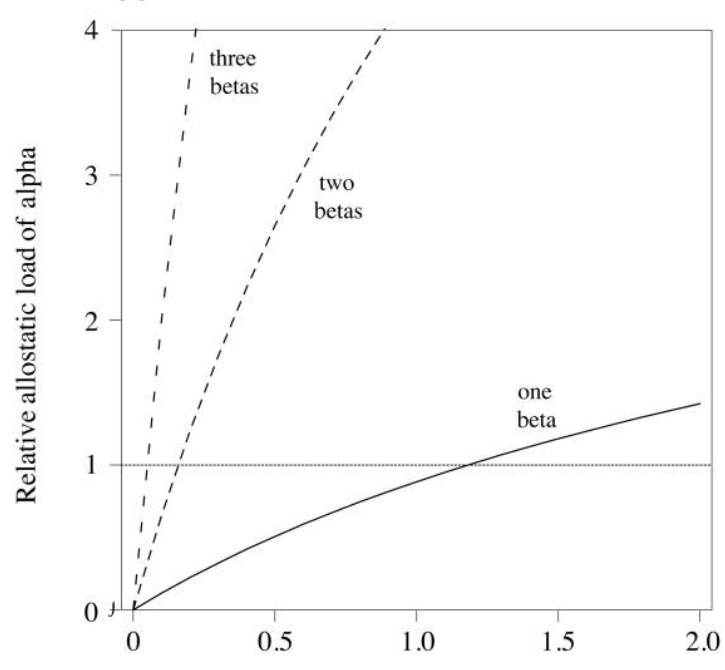

B

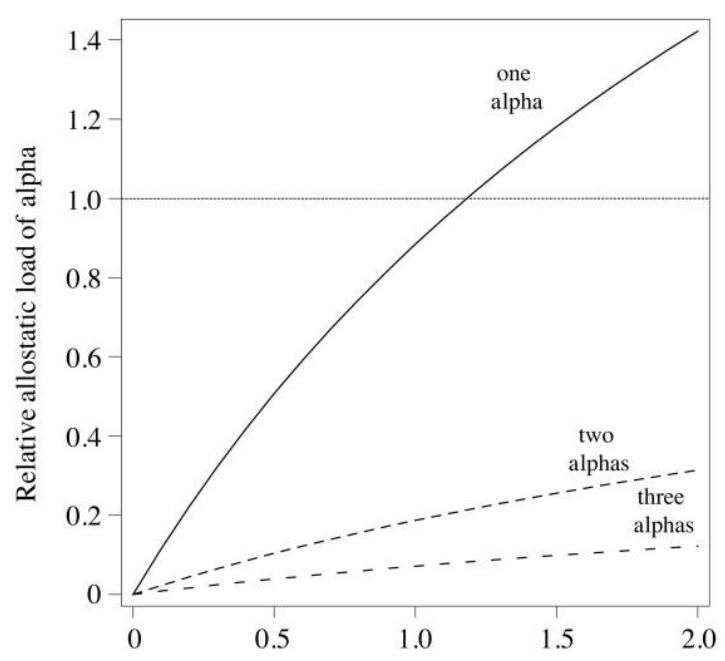

C

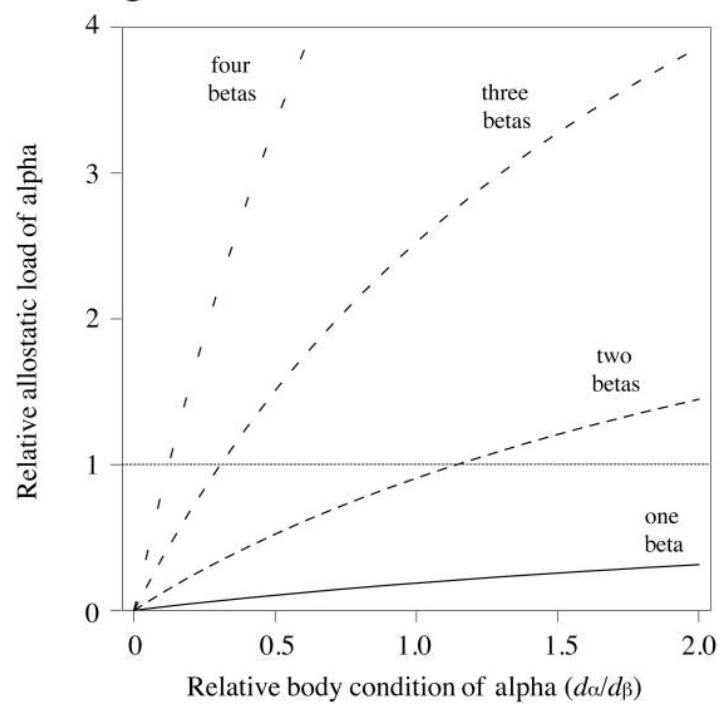

from 2002 to 2005 and captured male and female superb starlings from as many groups as possible during the prebreeding dry season. The prebreeding period was specifically chosen to sample corticosterone because (1) this is when reproductive roles are determined in this species, and (2) corticosterone would not be elevated because of the seasonal demands of reproduction (Rubenstein 2007a; Rubenstein et al. 2008). All field and laboratory methods are described in greater detail in the appendix, but they are briefly summarized here. We estimated the relative body condition of breeders as the ratio of the mean breeder standardized body mass (i.e., mass/tarsus residuals) to that of the corresponding subordinate standardized body mass in each group in each year of the study. Additionally, we estimated the relative allostatic load of breeders as the ratio of the mean baseline corticosterone of breeders to the mean level in helpers in each group in each year. Because we have shown previously that there are no sex differences in GC levels for this species (Rubenstein 2007a), data from males and females were combined for this study. Finally, because high nest predation during the egg stage precluded the identification of all helpers during the nestling stage (Rubenstein 2006), we were not able to accurately determine the number of helpers in each group. Instead, we used group size as a proxy for the ratio of alphas to betas in the group. Because the number of breeders in each group was fairly constant across years but the group size varied over time (Rubenstein 2007b), changes in group size reflected changes in the number of subordinates in the group. Thus, larger group sizes indicate smaller breeder-to-helper ratios, and vice versa.

We found that relative allostatic load was significantly related to group size and that there was a trend in the relationship between relative allostatic load and relative body condition (group size: $F_{1,10.72}=5.69, P=.037$; relative body condition: $F_{1,11.8}=3.65, P=.08$; interaction: $F_{1,11.92}=2.74, P=.12$; fig. 5); breeders had relatively higher allostatic loads in larger groups and when they were in relatively poorer body condition, and vice versa. Additionally, because samples sizes in each group were small and the variation in corticosterone values was high $(\mathrm{Ru}-$

Figure 4: Relative allostatic load of alpha in relation to relative body condition of alpha for $A$, groups with one alpha and a variable number of betas, $B$, one beta and a variable number of alphas, and $C$, two alphas and a variable number of betas. Relative allostatic load of alpha was calculated as the ratio of alpha's allostatic load to beta's allostatic load, and relative body condition of alpha was calculated as the ratio of alpha's body condition to beta's body condition. Solid and dashed lines indicate results from models with different numbers of group members. Dotted lines indicate a relative allostatic load for alpha of 1 . For all models, relatedness $(r)$ was 0.25 , the relative competitive ability $(b)$ was 0.8 , and the scaling factors $g, z$, and $e$ were $2,0.7$, and 0.3 , respectively. 


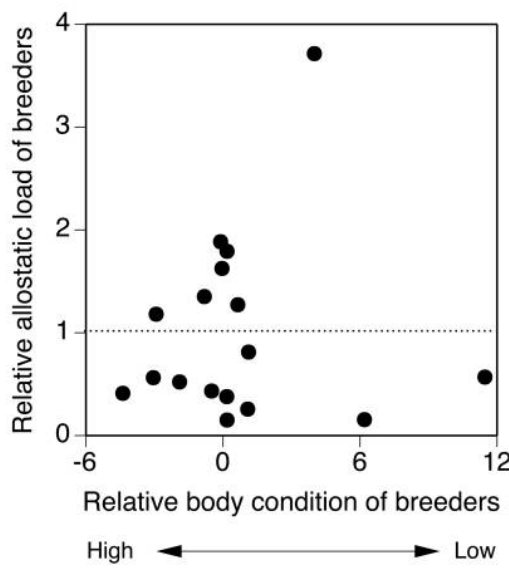

B

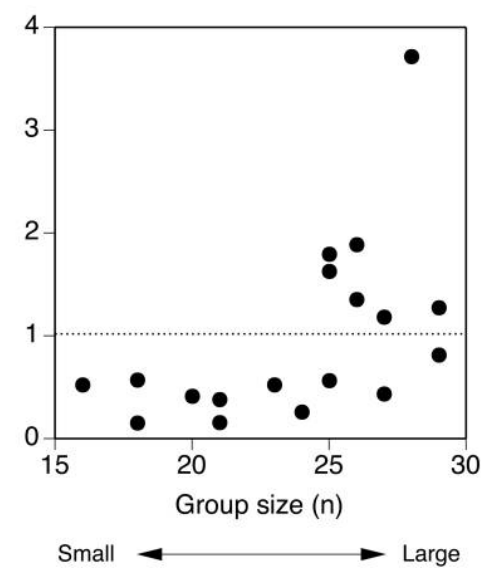

Figure 5: Relationship between relative allostatic load of breeders and $A$, relative body condition of breeders, and $B$, group size. Relative allostatic load of breeders was calculated as the ratio of breeder to helper baseline corticosterone in each group in each year of the study. Relative body condition of breeders was calculated as the ratio of breeder to helper standardized body mass in each group in each year of the study $(n=17$ groups). Group size was determined as the number of adult individuals in each group in each year ( $n=18$ groups). Dotted lines indicate a relative allostatic load of breeders of 1 .

benstein 2007a), we averaged values for each year of the study (vs. each group year) and found a significant relationship between relative allostatic load and relative body condition across years in our population $\left(F_{1,2}=23.28\right.$, $P=.04, r=0.96)$.

\section{Allostatic Load and the Costs of Social Status: From Models to Starlings}

Whereas initial interpretations of GC patterns in cooperatively breeding species suggested that it was more physiologically costly to be a dominant individual than a subordinate individual (Creel 2001), later comparative work showed that interspecific differences in GC levels were influenced by allostatic load or the relative costs of social status and dominance rank for each species (Goymann and Wingfield 2004); in some social species, it is more physiologically costly to be a dominant individual, but in others it is more costly to be a subordinate individual. We defined allostatic load following Goymann and Wingfield (2004), who described this personal cost as a product of both the aggressive effort expended and received over within-group social interactions. Thus, we built our allostatic load function to account for this combined effect of aggressive effort. We also specifically built our function to incorporate both social and environmental influences on allostatic load. To our knowledge, no study has considered the role that both environmental and social stress play in influencing GC levels, allostatic load, and social roles in cooperatively breeding vertebrates, despite the fact that allostatic load is influenced by both types of stressors (McEwen and Wingfield 2003).

We showed from model predictions and empirical data from cooperatively breeding superb starlings that body condition and group structure can dramatically affect allostatic load and are likely to explain both intra- and interspecific GC patterns in group-living animals. Both the two-player and the $n$-player versions of the model predicted that, under most conditions, subordinate individuals will always have higher allostatic loads than dominant individuals. Only when body condition is lower in dominant than subordinate individuals can the dominant individual experience higher allostatic load than the subordinate one. This suggests that dominant and subordinate individuals might respond or cope differently to environmental conditions. Empirical tests in superb starlings confirmed the expected relationship between relative body condition and relative GC levels; dominant breeders had higher GC levels when they were in poorer condition and lower GC levels when they were in better condition. Because this study and previous work in this system showed that there was no direct relationship between GC level and body condition in dominant breeders or subordinate helpers (Rubenstein 2007a), it is unlikely that the relationships observed here are simply due to nutritional stress leading to elevated GCs. Instead, the differential relationships are likely, at least in part, due to social influences on GC level or on allostatic load. Although further work is needed to elucidate the relationships between social stressors, environmental stressors, and GC levels in group-living species, 
it will be important to consider both types of stressors simultaneously in future studies of GCs in social vertebrates.

When our model was expanded from a two-player game to an $n$-player game where we varied the number of alphas and/or betas in the group, body condition was not the only important factor in influencing the relative allostatic load of alpha. Group structure also emerged as an important factor that explained why in some cases breeders might have higher allostatic loads than helpers, but in other cases helpers might have higher allostatic loads than breeders. That is, relative allostatic load of alphas increased as the number of betas in the group increased, but it decreased as the number of alphas in the group increased. Thus, alphas generally had higher allostatic loads than betas when there was a large number of betas in the group, but this cost could be reduced by increasing the number of alphas in the group. Again, testing this prediction in superb starlings confirmed the expected relationship between group size and relative GC level; dominant breeders had relatively higher GC levels as group size increased. Although we only tested the prediction by varying the number of helpers in the group, future studies should examine how varying the number of breeders in the group influences allostatic load.

Despite empirical support for the two key predictions generated from the model, there are limitations to both the model and our empirical test of the model. For example, although both social and environmental stressors appear to be important in influencing allostatic load and GC level, it is difficult to determine the relative importance of these two factors in shaping circulating hormones. Measuring circulating GCs will not simply provide a measure of the costs of group living. Instead, we must critically evaluate the link between GC level and allostatic load in different types of social vertebrate societies using controlled experiments and comparative studies. Additionally, superb starlings have a complex social system with large group sizes and helpers of both sexes. Testing the model predictions in simpler societies will inform us about the generality of the model. It is also important to note that we measured GCs during the nonbreeding season, when reproductive roles were being defined and GC levels were low. We caution that measuring GCs during the breeding season when their levels may be elevated-particularly in breeders-because of the seasonal demands of reproduction (Romero 2002) could confound results and interpretations. Nonetheless, the allostatic load model provides a robust framework within which to reevaluate existing GC data in social vertebrates and develop new studies to examine the costs of social status and dominance rank in group-living species.

\section{Allostatic Load and the Costs of Social Status: Other Vertebrate Societies}

Much of the published empirical data-particularly from social carnivores-shows that dominant individuals often have higher GC levels than subordinate individuals (Creel 2001, 2005; Sands and Creel 2004), which runs counter to much of the existing theory (Wasser and Barash 1983). We suggest that, in social carnivores and other species where dominant individuals have higher GC levels than subordinate individuals, either (1) differences in body condition between individuals of different social rank (e.g., dominant individuals might have poorer body condition than subordinate individuals) or (2) variation in group structure and dominance hierarchies (e.g., differences in reproductive sharing, differences in the number of breeders in a group, or the nature of the interactions between dominant individuals and subordinate individuals) are likely to explain why it may be more costly to be a dominant individual than a subordinate one. Although published data are not available from many species, limited data from meerkats Suricata suricatta support our conclusions and show that dominant individuals with lower body condition are less likely to evict subordinate individuals from the group than those with higher body condition (Young et al. 2006). In other words, reproductive conflict in meerkat groups is lower when the dominant individuals are in poorer condition, as our model predicts.

The predictions related to group structure have important implications for the evolution of group living in vertebrates. Because we found that the ratio of alphas to betas in the group was particularly important for influencing social costs, we hypothesize that the costs associated with group living (e.g., allostatic load) might (1) constrain group size in vertebrates, and (2) explain the formation of plural breeding societies (i.e., more than one dominant breeder; Brown 1978, 1987). If reproductive conflict and breeder allostatic load increase with increasing group size (i.e., number of subordinate helpers in the group), then the costs of group living might constrain group size by causing dominant breeders to cap the number of subordinate individuals allowed in the group. In other words, dominant individuals may be more likely to expel subordinate individuals from the group when a certain threshold number of subordinate individuals is reached. Additionally, when large groups confer greater reproductive benefits, dominant breeders might be able to reduce conflict and their own levels of allostatic load-while still maintaining larger group sizes and the associated benefits-by allowing other individuals to become breeders. However, understanding the relationships between the costs of group living, group size, and social structure requires determining how these costs are augmented by the 
fitness gains of group living (i.e., direct and indirect reproductive benefits). Although we considered only the costs of group living here, future studies that simultaneously examine the social costs and the reproductive benefits of sociality may inform us about the mechanisms underlying social diversity in group-living animals (Sherman et al. 1995). Additionally, modeling different types of hierarchies and accounting for greater within-role competition will make this model more applicable to a wider array of vertebrate societies.

\section{Conclusions and Future Directions}

The model presented here helps us to understand the underlying principles of how ecological and social factors affect the costs of living in social groups and how rank and social status affect levels of allostatic load in dominant and subordinate individuals. Our results demonstrate that two-player games are useful for making predictions that can be tested in complex animal societies; two-player games are not limited to systems with two individuals, but to systems with two distinct types of players (e.g., breeders and helpers). Scaling up from two-player to $n$-player reproductive skew models often changes the predictions of the models (e.g., Johnstone et al. 1999; Reeve and Emlen 2000; but see Reeve and Jeanne 2003). However, in this study, increasing the number of alphas and/or betas does not qualitatively change the results. That is, there still exists a parameter space where alphas have higher allostatic loads than betas, and vice versa. The $n$-player version of the model shows that both body condition and group structure can be important in influencing the costs of social status. These results may help us to interpret both intra- and interspecific patterns of GCs in social vertebrates. Moreover, these results suggest that the costs of dominance rank will vary greatly between singular and plural cooperatively breeding species where group sizes and the numbers of breeders in the groups differ, and that examining how allostatic load varies in species with different types of dom inance hierarchies may help to explain alternative routes to the evolution of sociality in animals.

Examining the factors that influence reproductive conflict in cooperatively breeding species is vital for understanding both the causes and the consequences of group living. Because overt aggression can be rare or difficult to observe in many social vertebrates, measuring GC stres: $\rightarrow$ hormones may allow researchers to quantify relative levels of aggression and examine the costs associated with sociar status and dominance rank (i.e., allostatic load). Clearly, there is work to be done to further elucidate the relation. ships between GCs, dominance rank, and allostatic load. However, the concept of allostatic load has further utility for studying the evolution of sociality in vertebrates be- cause it synthesizes how environmental and social factors influence reproductive behavior and physiology and ultimately drive individual reproductive decisions (McEwen and Wingfield 2003; Goymann and Wingfield 2004; Rubenstein $2007 a$ ). Reproductive skew theory, which by definition focuses on reproductive sharing and the benefits of cooperative group living, has provided a theoretical framework to help understand the evolution of cooperation in animals (e.g., Emlen 1999; Johnstone 2000). We have expanded this framework by considering the personal costs of social status and dominance rank, and we have provided the first theoretical model of the larger concept of allostasis (i.e., physiological stability through social and environmental change), an important emerging concept in behavioral neuroendocrinology that links mechanistic processes with ultimate causation (McEwen and Wingfield 2003). As these types of models are expanded to account for social groups with different types of dominance hierarchies, they will become increasingly useful for understanding how reproductive conflict influences the costs of dominance rank and how GCs can be used to study cooperation and conflict in group-living vertebrates.

\section{Acknowledgments}

We thank S. Flaxman, K. Reeve, and D. I. Rubenstein for constructive feedback on this manuscript. We acknowledge the Kenyan Ministry of Education, Science, and Technology, the National Museums of Kenya Ornithology Department, the Kenya Wildlife Service, and the Mpala Research Centre for enabling work in Kenya. D.R.R. was supported by a fellowship from the Miller Institute for Basic Research in Science at the University of California, Berkeley. S.-F.S. acknowledges the Department of Neurobiology and Behavior at Cornell University and the Cornell Laboratory of Ornithology for continued support.

\section{Literature Cited}

Brown, J. L. 1978. Avian communal breeding systems. Annual Review of Ecology and Systematics 9:123-155.

- 1987. Helping and communal breeding in birds: ecology and evolution. Princeton University Press, Princeton, NJ.

Buchanan, K. L., K. A. Spencer, A. R. Goldsmith, and C. K. Catchpole. 2003. Song as an honest signal of past developmental stress in the European starling (Sturnus vulgaris). Proceedings of the Royal Society B: Biological Sciences 270:1149-1156.

Charnov, E. L., and J. R. Krebs. 1974. On clutch-size and fitness. Ibis 116:217-219.

$\rightarrow$ Cockburn, A. 1998. Evolution of helping behaviour in cooperatively breeding birds. Annual Review of Ecology and Systematics 29:141177.

Creel, S. 2001. Social dominance and stress hormones. Trends in Ecology \& Evolution 16:491-497.

. 2005. Dominance, aggression, and glucocorticoid levels in social carnivores. Journal of Mammalogy 86:255-264. 
Creel, S., and N. M. Creel. 2002. The African wild dog: behavior, ecology, and conservation. Princeton University Press, Princeton, NJ.

$\rightarrow$ Cuthill, I. C., S. A. Maddocks, C. V. Weall, and E. K. M. Jones. 2000. Body mass regulation in response to changes in feeding predictability and overnight energy expenditure. Behavioral Ecology $11-$ 189-195.

$\rightarrow$ Emlen, S. T. 1982. The evolution of helping. II. The role of behavioral conflict. American Naturalist 119:40-53.

$\rightarrow-$. 1995. An evolutionary theory of the family. Proceedings of the National Academy of Sciences of the USA 92:8092-8099.

. 1999. Reproductive skew in cooperatively breeding birds: ar overview of the issues. Pages 2922-2931 in Proceedings of the 22nd International Ornithological Congress.

Faulkes, C. G., and D. H. Abbott. 1997. The physiology of reproductive dictatorship: regulation of male and female reproductior by a single breeding female in colonies of naked mole-rats. Pages 302-334 in N. G. Solomon and J. A. French, eds. Cooperative breeding in mammals. Cambridge University Press, Cambridge.

$\rightarrow$ Frank, S. A. 1995. Mutual policing and repression of competition in the evolution of cooperative groups. Nature 377:520-522.

$\rightarrow$ Goymann, W., and J. C. Wingfield. 2004. Allostatic load, social status and stress hormones: the costs of social status matter. Anima Behaviour 67:591-602.

$\rightarrow$ Hamilton, W. D. 1964. Genetical evolution of social behaviour. Journal of Theoretical Biology 7:1-16.

$\rightarrow$ Jakob, E. M., S. D. Marshall, and G. W. Uetz. 1996. Estimating fitness $\rightarrow$ a comparison of body condition indices. Oikos 77:61-67.

$\rightarrow$ Johnstone, R. A. 2000. Models of reproductive skew: a review and synthesis. Ethology 106:5-26.

$\rightarrow$ Johnstone, R. A., R. Woodroffe, M. A. Cant, and J. Wright. 1999. Reproductive skew in multimember groups. American Naturalist 153:315-331.

$\rightarrow$ Maynard Smith, J., and G. A. Parker. 1976. The logic of asymmetri $\rightarrow$ contests. Animal Behaviour 24:159-175.

$\rightarrow$ Mays, N. A., C. M. Vleck, and J. Dawson. 1991. Plasma luteinizing hormone, steroid hormones, behavioral role, and nest stage in cooperatively breeding Harris hawks (Parabuteo unicinctus). Auk 108:619-637.

$\rightarrow$ McEwen, B. S., and J. Wingfield. 2003. The concept of allostasis in biology and biomedicine. Hormones and Behavior 43:2-15.

$\rightarrow$ Parker, G. A., and D. I. Rubenstein. 1981. Role assessment, reserve strategy, and acquisition of information in asymmetric animal con flicts. Animal Behaviour 29:221-240.

$\rightarrow$ Reeve, H. K., and S. T. Emlen. 2000. Reproductive skew and group size: an $N$-person staying incentive model. Behavioral Ecology $11-$ 640-647.

$\rightarrow$ Reeve, H. K., and R. L. Jeanne. 2003. From individual control to majority rule: extending transactional models of reproductive skew in animal societies. Proceedings of the Royal Society B: Biologica Sciences 270:1041-1045.

$\rightarrow$ Reeve, H. K., and L. Keller. 2001. Test of reproductive-skew models in social insects. Annual Review of Entomology 46:347-385.

$\rightarrow$ Reeve, H. K., and S.-F. Shen. 2006. A missing model in reproductive skew theory: the bordered tug-of-war. Proceedings of the National Academy of Sciences of the USA 103:8430-8434.

$\rightarrow$ Reeve, H. K., S. T. Emlen, and L. Keller. 1998. Reproductive sharing in animal societies: reproductive incentives or incomplete control by dominant breeders? Behavioral Ecology 9:267-278.

$\rightarrow$ Romero, L. M. 2002. Seasonal changes in plasma glucocorticoid con- centrations in free-living vertebrates. General and Comparative Endocrinology 128:1-24.

Rubenstein, D. R. 2006. The evolution of the social and mating systems of the plural cooperatively breeding superb starling, Lamprotornis superbus. PhD diss. Cornell University, Ithaca, NY.

-2007a. Stress hormones and sociality: integrating social and environmental stressors. Proceedings of the Royal Society B: Biological Sciences 274:967-975.

$\rightarrow-$. 2007b. Temporal but not spatial environmental variation drives adaptive offspring sex allocation in a plural cooperative breeder. American Naturalist 170:155-165.

Rubenstein, D. R., A. F. Parlow, C. R. Hutch, and L. B. Martin. 2008. Environmental and hormonal correlates of immune activity in a cooperatively breeding tropical bird. General and Comparative Endocrinology 159:10-15.

Sands, J., and S. Creel. 2004. Social dominance, aggression and faecal glucocorticoid levels in a wild population of wolves, Canis lupus. Animal Behaviour 67:387-396.

Schoech, S. J., R. L. Mumme, and M. C. Moore. 1991. Reproductive endocrinology and mechanisms of breeding inhibition in cooperatively breeding Florida scrub jays (Aphelocoma c. coerulescens). Condor 93:354-364.

Schoech, S. J., R. L. Mumme, and J. C. Wingfield. 1997. Corticosterone, reproductive status, and body mass in a cooperative breeder, the Florida scrub jay (Aphelocoma coerulescens). Physiological Zoology 70:68-73.

$\rightarrow$ Sherman, P. W., E. A. Lacey, H. K. Reeve, and L. Keller. 1995. The eusociality continuum. Behavioral Ecology 6:102-108.

Solomon, N. G., and J. A. French. 1997. Cooperative breeding in mammals. Cambridge University Press, Cambridge.

Stacey, P. B., and W. D. Koenig. 1990. Cooperative breeding in birds: long-term studies of ecology and behavior. Cambridge University Press, Cambridge.

Taylor, P. D., and S. A. Frank. 1996. How to make a kin selection model. Journal of Theoretical Biology 180:27-37.

Trivers, R. L. 1972. Parental investment and sexual selection. Pages 136-179 in B. Campbell, ed. Sexual selection and the descent of man 1871-1971. Aldine, Chicago.

$\rightarrow$ Vehrencamp, S. L. 1983a. A model for the evolution of despotic versus egalitarian societies. Animal Behaviour 31:667-682.

$\rightarrow$. 1983b. Optimal degree of skew in cooperative societies. American Zoologist 23:327-335.

$\rightarrow$ Wasser, S. K., and D. P. Barash. 1983. Reproductive suppression among female mammals: implications for biomedicine and sexual selection theory. Quarterly Review of Biology 58:513-538.

$\rightarrow$ Wingfield, J. C., R. E. Hegner, and D. M. Lewis. 1991. Circulating levels of luteinizing hormone and steroid hormones in relation to social status in the cooperatively breeding white-browed sparrow weaver, Plocepasser mahali. Journal of Zoology 225:43-58.

Witter, M. S., J. P. Swaddle, and I. C. Cuthill. 1995. Periodic food availability and strategic regulation of body mass in the European starling, Sturnus vulgaris. Functional Ecology 9:568-574.

$\rightarrow$ Young, A. J., A. A. Carlson, S. L. Monfort, A. F. Russell, N. C. Bennett, and T. Clutton-Brock. 2006. Stress and the suppression of subordinate reproduction in cooperatively breeding meerkats. Proceedings of the National Academy of Sciences of the USA 103: 12005-12010.

Associate Editor: Greg Demas Editor: Monica A. Geber 